\title{
Publisher's Note: Quantum Speed Limits for Leakage and Decoherence [Phys. Rev. Lett. 115, 210402 (2015)]
}

Iman Marvian and Daniel A. Lidar

(Received 23 November 2015; published 8 December 2015)

DOI: 10.1103/PhysRevLett.115.249902

PACS numbers: 03.67.Ac, 03.65.Yz, 03.67.Lx, 03.67.Pp, 99.10.Fg

This paper was published online on 18 November 2015 with an omission in the Acknowledgments. The Acknowledgments should read as "This Letter was supported under ARO MURI Grants No. W911NF-12-1-0541, NSF No. CCF-1254119, ARO Grant number W911NF-12-1-0523, and ARO MURI Grant No. W911NF-11-1-0268. We thank P. Zanardi, S. Lloyd, and E. Farhi for useful discussions." The Acknowledgments have been corrected as of 20 November 2015. The Acknowledgments are correct in the printed version of the journal. 